\title{
Corruption et le déclin de la République romaine
}

Par Guillaume Jasmin

\begin{abstract}
This paper takes a look at the role of corruption in ending the Roman Republic. It does not purport to hold up corruption as the single deciding factor in the Roman Republic's collapse but rather hopes to show that corruption did have a responsibility in bringing down a 500 year-old institution. This dissertation was written by reviewing existing sources be they historical or analytical so as to find the elements needed to come to a conclusion answering the question posed above. The structure followed by this paper is as follows: the author first exposed how the Roman Republic's political system was organized and how it functioned. Secondly, the author defined corruption and detailed a historical example of this insidious entity. Finally, the fall of the Republic is chronicled and the role of corruption in this collapse is uncovered. To conclude, the author found that corruption did in fact play a non-negligible role in the overthrow of the Roman Republic.
\end{abstract}

Résumé: Cette dissertation essaye de présenter quel a été le rôle de la corruption dans la chute de la République romaine. Toutefois, ce texte n'essaye pas d'assigner un rôle décisif à la corruption, mais tente plutôt de montrer que la corruption (parmi plusieurs autres facteurs) avait une responsabilité dans l'effondrement d'une institution vieille de plus de 500 ans. Cette rédaction a été écrite en révisant plusieurs sources historiques et analytiques existantes afin de trouver les éléments qui ont servis à répondre à la question ci-dessus. La structure de cette dissertation va comme suit: premièrement, l'auteur a exposé l'organisation et le fonctionnement de l'appareil politique qu'était la République romaine. Puis, l'auteur a définit la corruption et détaillé un exemple historique de cette entité insidieuse. Ensuite, l'auteur a chroniquer l'écroulement de la République et a mis à jour le rôle de la corruption dans cet affaissement. En conclusion, l'auteur à découvert que la corruption a joué un rôle non-négligeable dans la destruction de la République romaine.

Nous ne pouvons sous-estimer l'importance de l'époque romaine pour l'histoire humaine. Cette époque continue de nous fasciner et a eu de très grandes répercussions culturelles, religieuses, politiques et économiques sur l'Europe et le monde. Très souvent, nous oublions que l'époque romaine ne se compose pas uniquement des légions de César, des guerres puniques, d'empereurs tels que Néron, Auguste, Hadrien ou Trajan ou de la tombée de l'Empire. Notre imaginaire est rempli d'image de gladiateurs, d'orgies, de toges et de dieux tels que Jupiter, Minerva, Mars et Mercure. Nous avons tendance à nous arrêter sur certains grands points sans chercher à en connaître plus. Nous devons cependant nous rappeler que l'époque romaine est divisée en trois époques : la royauté romaine de 753-509 avant Jésus Christ, l'Empire romain de 27 avant Jésus Christ jusqu'à 1453 après Jésus Christ, date à laquelle la partie est de l'Empire s'écroule (la partie ouest de l'Empire s'était effondré bien avant, en 476) et la République (50927 av. J. C.) qui se trouve entre les deux périodes ci-dessus. C'est de la période de la République 
que traitera ce texte. Un auteur de cette époque, Marcus Tullius Cicero (dit Cicéron), nous sera indispensable afin d'étudier l'ère de la République.

Né en 106 avant Jésus Christ et assassiné en 43 de la même période, Cicéron fait figure de proue parmi les plus grands philosophes, orateurs, politiciens, hommes d'état et théoriciens politique romains de son époque. Ses idées politiques ainsi que le fonctionnement de la République sont très accessibles par l'entremise de ses nombreux écrits tels que De la République, Des Lois et le Traité des Devoirs. Cependant, cette dissertation ne traitera ni de l'origine du pouvoir, ni de son organisation, ni de son exercice, bien que ces éléments feront partie de la discussion. Cette dissertation portera plutôt sur le phénomène insidieux qu'est la corruption. Ayant occupé les positions les plus importantes (questeur, édile, préteur et consul) au sein de la République, Cicéron nous offre, non seulement ces écrits politiques, mais nous savons aussi qu'il a été témoin de la corruption (politique, économique, morale, etc.) En fait, en tant qu'avocat, Cicéron a défendu plusieurs haut placés romains contre des accusations de corruption et d'extorsion et a aussi agit en tant que procureur lors du procès pour extorsion du gouverneur de Sicile, Caius Verres, le seul procès où Cicéron aurait été procureur. ${ }^{1}$ Ainsi, Cicéron est un auteur bien désigné pour ce travail qui cherche à comprendre la corruption à l'époque républicaine. Puisque Cicéron a vécu vers la fin du régime républicain il est possible qu'il puisse aussi nous offrir des pistes quant à la chute de ce régime et le rôle de la corruption (si c'est le cas) dans cette chute.

Le texte suivant se penchera sur trois différents aspects afin d'évaluer les impacts de la corruption. Il y aura premièrement une exposition de l'organisation et du fonctionnement politique romain à l'époque de la République et de Cicéron. Ensuite, viendra une discussion de ce qu'est exactement la corruption, sa définition chez les Romains, ses différentes formes et où elle s'insère dans la politique républicaine. Ces deux premières sections permettront à l'auteur d'établir le contexte de l'époque et définir certains concepts. Puis, pour terminer, nous en viendrons aux effets de la dite corruption, afin de vérifier si celle-ci a contribué à l'affaissement de la République et à l'avènement de l'Empire.

\section{La République}

Pour comprendre la corruption et son rôle dans la République, il est primordial de premièrement comprendre l'organisation et le fonctionnement de celle-ci. Nous ne pouvons comprendre quelque chose qui afflige un système politique sans préalablement comprendre ce système. Cicéron nous servira d'exemple afin d'illustrer comment un homme politique gravissait les échelons.

\section{Organisation du système politique}

L'organisation de la Rome républicaine reposait fortement sur un ensemble de magistratures. Non seulement y avait-il plusieurs positions mais aussi plusieurs lois et exceptions ainsi que des distinctions entre position patriciennes et plébéiennes. Néanmoins, ces positions étaient très importantes et très recherchées. Il y avait quatre grandes positions : les questeurs, les édiles, les prêteurs et les consuls. De plus, les plébéiens avaient aussi instauré leurs propres positions soient les tribuns. Il y avait aussi le Concile de la plèbe ainsi que le Sénat.

1Fuhrmann, Manfred. Cicero and the Roman Republic translated by W.E. Yuill. Cambridge Mass.: Blackwell Publishers Ltd., 1995, 42. 
Les quatre grandes magistratures formaient un ensemble qui était appelé le cursus honorum. Il s'agissait plus ou moins de l'échelle politique que l'on devait gravir. Le premier échelon, la questure, fût instaurée afin de s'occuper des finances publiques et cette position était accessible à partir de l'âge de 28 ans. ${ }^{2}$ Les questeurs pouvaient tout aussi bien se retrouver dans des positions administratives au sein du Trésor de Rome que dans les provinces comme assistants aux gouverneurs des provinces. Cicéron, lui-même fût élu questeur en 76 et servit auprès du gouverneur Sextus Peducaeus en Sicile. ${ }^{3}$

Le deuxième échelon était celui d'édile. Ceux-ci étaient «chargés d'exécuter les principales dépenses publiques : organisation des Jeux par les édiles; mise à ferme des travaux et fournitures par les censeurs.» ${ }^{4}$ Ainsi, les édiles et les censeurs s'occupaient plus de la ville de Rome. Ils travaillaient ensemble afin d'assurer le bon fonctionnement de la ville. Murell écrit en reprenant Cicéron que celui-ci comprenait l'importance de ce poste, en assurant qu'il mettrait sur pied de grandes fêtes et jeux pour le peuple, qu'il prendrait soin des temples sacrés et protègerait la cité. ${ }^{5}$ Cicéron fût élu édile en 69.

Le troisième échelon du cursus honorum était la position de préteur, au nombre de six. Ceux-ci s'occupaient surtout des lois et de l'administration de la justice et était plus ou moins indépendants. ${ }^{6}$ Grimal écrit «...deux assuraient le fonctionnement de la justice; l'un le préteur urbain, entre les citoyens; l'autre, le préteur pérégrin, dans les procès où l'une des deux parties était un étranger. Les quatre autres étaient envoyés en mission : commandement d'une armée ou d'une flotte, gouvernement d'une province.» ${ }^{7}$ Cicéron fût élu au poste de préteur en 66.

La dernière position (aussi la plus prestigieuse) était celle de consul. Celles-ci étaient au nombre de deux et s'étaient départagés le pouvoir royal. Ainsi, l'une des positions exerçait l'imperium ou l'autorité militaire et civile et l'autre exerçait les auspices. Ces positions alternaient le pouvoir à chaque mois et était en place pendant un an. Cicéron fût élu consul en $63 .^{8}$

Le Sénat était composé des plus hauts membres de la plèbe, connus sous le nom de patricien, des anciens magistrats ainsi que des magistrats au pouvoir. Ces gens élisaient les consuls. Ils donnaient aussi leurs avis et votaient sur les décisions populaires. Les plébéiens avaient eux aussi leurs magistratures et leur concile. Après toutes les réformes, les plébéiens finirent par se doter d'un concile qui élisaient les tribuns de la plèbe (représentant de la population) et les édiles plébéiens. Les tribuns avaient comme pouvoir d'intercéder en faveur de la population et pouvaient opposer les décisions du Sénat, des consuls etc. De plus, le concile de la plèbe votait les plébiscites et jugeait ses opposants. ${ }^{9}$

${ }^{2}$ Combès, Robert. La République à Rome (509-29 av. J.-C.). Paris, France : Presses Universitaires de France, 1972, 135-136.

${ }^{3}$ Murell, John. Cicero and the Roman Republic. Cambridge, U.K.: Cambridge University Press, $2008,33$.

4 Combès, La République à Rome, 136.

5 Murell, Cicero and the Roman Republic, 43.

${ }^{6}$ Combès, La République à Rome, 134.

${ }^{7}$ Grimal, Pierre. La civilisation romaine. Paris, France: Les Éditions Arthaud, 1984, 116.

${ }^{8}$ Maillet-Rao, Caroline. "La royauté et la république romaine" Notes de cours, Science Sociales 311, Automne 2011.

${ }_{9}^{9}$ Maillet-Rao, Caroline. "La royauté et la république romaine" Notes de cours, Science Sociales 311, Automne 2011. 
Finalement, il y avait une magistrature extraordinaire qui pouvait être mis en place si l'on jugeait que la situation interne mettait en péril la République : le dictateur. Celui-ci était nommé par un consul, disposait d'un pouvoir limité à 6 mois (mais un pouvoir plus grand que tout autre magistrat) et avait la tâche de sauver la République. ${ }^{10}$ Nous verrons que cette position aura un grand rôle vers la fin de République.

Ceci conclut notre survol rapide des magistratures et institutions importantes de la République. La prochaine section se concentrera sur comment le système politique républicain fonctionne, en portant un regard particulier sur le processus électoral et l’organisation des élections.

\section{Fonctionnement du système politique}

Les magistratures décrites ci-dessus étaient toutes très importantes au bon fonctionnement de la République. Certaines s'occupaient des finances, d'autres de la justice et des lois. Mais, nous devons aussi comprendre comment celles-ci étaient élues et comment le système fonctionnait.

Pour commencer, nous devons exposer comment les votes étaient répartis. Donc, les comices centuriates (le peuple) votaient pour les magistrats supérieurs et les comices tributes votaient pour les magistrats inférieurs. ${ }^{11}$ Les comices centuriates rassemblaient toute la plèbe puis les répartissaient en centuries. Celles-ci étaient des organes militaires puis ont peu à peu pris un sens politique et électoral, car chaque centurie recevait un vote au sein des élections. Les centuries étaient aussi organisées en fonction de la richesse, les plus riches étant capable de s'acheter tout l'accoutrement nécessaire pour faire la guerre et les plus pauvres non. ${ }^{12}$

Il y avait ainsi 18 centuries équestres, composées des hommes les plus riches, ceux qui avaient assez d'argent pour se procurer des armes, des armures ainsi qu'un cheval. Ensuite venait 80 centuries d'hoplites, ces hommes qui pouvaient se procurer armes et armures mais pas de cheval. Les 20 centuries suivantes se composaient d'hommes qui ne possédaient pas de cuirasses, 20 autres qui ne possédaient ni cuirasse ni jambière, 20 centuries qui n'avaient que des javelots et des lances et 30 qui se battaient avec des frondes. Puis, il existait aussi 7 centuries qui ne faisaient pas partie de celles décrites plus haut. Il y avait une centurie d'anciens consuls issue des patriciens, deux centuries de musiciens, deux centuries d'ouvriers, une de gens qui, comme des vautours, attendaient que quelqu'un meurt pour s'emparer de ses armes afin de se battre et enfin une centurie de prolétaire, ces gens qui n'avaient absolument rien à offrir autre que leur labeur et qui représentaient environ la moitié de la population. ${ }^{13}$

Lorsque venait le temps des élections, l’armée se rassemblait sur le Champ de Mars et on procédait au choix de la centuria praerogativa, la centurie prérogative, la première à voter, puis on procédait avec la liste débutant avec les centuries équestres. Gaillard explique que le vote de la centurie prérogative était crucial car elle présageait la nature et

\footnotetext{
10 Klein, Bernard. "Chapitre 1 - Rome, des origines à la fin du IIIe siècle." Dans Le monde romain : histoire ancienne, sous la direction de M. Kaplan, 11-46. Rosny, France: Bréal, 1995, 32.

11 Gaillard, Jacques. "Les rituels politiques de la République." Dans Rome $1^{\text {er }}$ siècle av. J.-C. Ainsi périt la République des vertus..., dirigé par Jacques Gaillard, 42-57. Paris, France : Les Éditions Autrement, $1996,46$.

12 Maillet-Rao, "La royauté et la république romaine" Notes de cours Science Sociales 311, Automne 2011.

13 Maillet-Rao, "La royauté et la république romaine" Notes de cours Science Sociales 311, Automne 2011.
} 
la direction du vote, puisque les centuries suivantes, plus souvent qu'autrement, votait comme elle. ${ }^{14}$ Sur les 193 centuries (d'autres diront 195, peu importe), les centuries riches (18 équestres, 80 hoplites) détenaient donc la majorité. Lorsque la majorité était atteinte, on arrêtait le vote et les centuries plus pauvres (bien qu'elles représentaient la majorité de la population) n'avaient jamais leur mot à dire.

Maintenant que nous avons vu comment fût organisée la République et comment celle-ci fonctionnait, nous pouvons nous concentrer sur la corruption en elle-même.

\section{La République corrompue}

La corruption est un phénomène fourbe qui se retrouve partout au monde, sans exception. Elle infiltre et pourrit tout ce qu'elle touche. La République romaine n'était aucunement exempte de ceci. Cette partie examinera comment est définie la corruption et quelles formes celle-ci prend.

La corruption : sa définition et ses formes

Lòpez nous signale premièrement la difficulté de définir la corruption puisque c'est un phénomène des plus complexes. Malgré ces difficultés nous avons été en mesure d'établir des principes qui indiquent ce qu'est la corruption. Lòpez reprend Seña en écrivant que « la corruption implique une violation des devoirs d'une charge ou de ceux imposés par l'État; Elle implique un bénéfice privé, mais pas nécessairement d’ordre pécunier; Elle se fait toujours au sein d'un système normatif qui approuve ou condamne l'action d'un point de vue légal, social ou éthique. »15 À la même page, Lòpez continue en notant que la corruption dans la Républicaine romaine entre dans ses trois catégories puisque la corruption allait à l'encontre des lois, était commise dans l'exercice de charges et fonctions publiques et renflouait les coffres des auteurs de ces crimes.

Un peu plus loin, l'auteure démontre que les Romains avaient en fait une loi contre la corruption : «Il [le magistrat] ne doit pas s'emparer ou accepter de cadeau, de récompense, de salaire ou d'autre chose aux frais de l'État ou en raison d'une charge publique,...; ni rien faire pour qu'une telle chose revienne à lui-même ou à son personnel. »16 Il est donc clair que les magistrats ne pouvaient recevoir quoi que ce soit. De plus, Lòpez reprend Cicéron ${ }^{17}$ en écrivant que lui-même condamnait ces pratiques, croyant celles-ci immorales, honteuses, criminelles et infâmes. Lòpez finit son exposé en montrant que les Romains considéraient plusieurs choses comme illicites, entre autres :

«L'appropriation illégitime de biens, propriétés de l'État ou de particuliers; et l'acceptation de gratifications illégales. »18

Ainsi, il est évident que la corruption était bel et bien présente durant la République romaine mais aussi que les Romains savaient qu'elle causait des ravages. Passons maintenant aux différentes formes que la corruption prenait aux temps des Romains.

\footnotetext{
${ }^{14}$ Gaillard, Jacques. "Les rituels politiques de la République." Dans Rome $1^{\text {er }}$ siècle av. J.-C. Ainsi périt la République des vertus..., dirigé par Jacques Gaillard, 42-57. Paris, France : Les Éditions Autrement, $1996,47$.

15Rosillo López, Cristina. La corruption à la fin de la République romaine (IIe-Ier s. av. J.-C.) : Aspects politiques et financiers. Stuttgart, Allemagne : Franz Steiner Verlag, 2010, 17.

16 Idem, 19.

${ }^{17}$ Rosillo López, Cristina. La corruption à la fin de la République romaine (IIe-Ier s. av. J.-C.) : Aspects politiques et financiers. Stuttgart, Allemagne : Franz Steiner Verlag, 2010, 20.

18 Idem, 23.
} 
Premièrement, examinons la corruption électorale. Comme nous l'avons vu plus haut, les élections étaient très importantes quant au fonctionnement du système républicain. Elles jouaient un rôle suprêmement important et les élections devenaient ainsi une cible parfaite pour les gens cherchant à corrompre le système. Il ne suffisait que d'influencer les électeurs afin de s'assurer leur vote. Lacey écrit : «Since the Roman assemblies decided questions by the votes of a majority of voting-units based on the tribes, there had risen a class of men who distributed to the members of their own tribe the money for bribes received from candidates for election. In this way a candidate could secure the votes of enough tribes to win success. " Ainsi, on voit que des gens prenaient l'argent des pots-de-vin qui venaient des candidats et redistribuaient le tout au sein de la tribut afin d'assurer que les électeurs élisent celui qui leur avait verser la « donation » ${ }^{19}$. Lòpez indique une pratique similaire qui visait la centuria praerogativa, la première centurie à voter lors des élections. Nous avons vu ci-haut, qu'il était commun que les centuries votant après celle-ci suivent son exemple et votent pour le même candidat. Donc, les candidats offraient beaucoup d'argent à la centuria praerogativa pour que celle-ci votent pour eux et non pour les autres. Ils pouvaient, en quelque sorte, contrôler les élections. ${ }^{20}$ Ceci ne représente que deux exemples de corruption électorale parmi plusieurs.

La deuxième forme est la corruption politique. Selon Lòpez, celle-ci peut être divisée en deux groupes distincts : le péculat ou le vol à l'État (objets et argents) et l'extorsion. ${ }^{21}$ Les deux crimes sont commis par un magistrat qui occupe une position publique et qui utilise cette position à mauvais escient. La troisième et dernière forme de corruption que ce texte examinera est la corruption judiciaire. Celle-ci revient, plus ou moins, à donner des sommes d'argent faramineuses aux juges afin de s'assurer d'un verdict. La prochaine partie, celle qui traitera de Caius Verres, nous permettra d'aller en plus de détails sur ces deux formes.

Nous devons aussi signaler le fait que les trois configurations de la corruption n'étaient et ne sont aucunement autonomes. Le plus souvent l'une servait les autres et viceversa. Par exemple, pour pouvoir payer les pots-de-vin en cas de corruption électorale ou de corruption judiciaire, les magistrats doivent se procurer l'argent d'une manière ou d'une autre. C'est ici qu'entre la corruption politique car en extorquant les provinces ou en cambriolant les coffres de l'État, les magistrats amassent des fonds afin de perpétrer leurs crimes. Un autre exemple est celui d'un magistrat qui paye des juges afin d'éliminer un adversaire politique.

Comme il a été mentionné plus haut, la prochaine section traitera d'un exemple concret de la corruption et du rôle de Cicéron comme procureur lors de cette cause.

\section{Caius Verrès, gouverneur de Sicile}

\footnotetext{
${ }^{19}$ Lacey, W. K. Cicero and the end of the Roman Republic. Londres, U.K.: Hodder and Stoughton, 1978, $23-24$.

${ }^{20}$ Rosillo López, La corruption à la fin de la République romaine (IIe-Ier s. av. J.-C.) : Aspects politiques et financiers, 17.

${ }^{21}$ Idem, 88 et 96 respectivement.
} 
Caius Verrès fût gouverneur de Sicile de 73 à 71 avant Jésus-Christ. Durant son règne, on l'accusa de corruption, plus précisément de péculat ainsi que d'extorsion. Murrell reprenant Cicéron écrit que durant son gouvernement, Verrès avait empoché les profits de la première année, qu'il avait distribuer les profits de la deuxième année à ses patrons et aux gens qui le défendait et que les profits de la troisième année avait été réservés aux juges. ${ }^{22}$ Ainsi, on a des preuves que Verrès commettait de la corruption politique mais aussi de la corruption judiciaire. En 69 avant J.-C., avant le début du procès, Cicéron fût élu édile. Mais le chemin fût semé d'embûches. Verrès essaya d'utiliser l'argent extorqué aux Siciliens pour essayer de bloquer l'élection de Cicéron. ${ }^{23}$ Donc, Verrès était aussi coupable de corruption électorale. Ceci nous permet aussi de retourner à ce qui a été dit plus haut par rapport aux formes de corruption qui travaille ensemble. L'argent que Verrès avait extorqué avait été utilisé à des fins de corruption électorale et judiciaire.

Finalement, Cicéron fût choisi comme procureur lors de ce procès, mais, une fois de plus, ceci ne s'est pas fait sans embûches. Des copains de Verrès essayaient de se faire élire édile, questeur et prêteur afin de limiter les dégâts et d'empêcher Cicéron de mener à bien ses enquêtes. Verrès utilisa l'argent qu'il avait extorqué afin d'offrir des pots-de-vin au gens pour que ceux-ci ne témoignent pas. ${ }^{24}$ Pourtant, Cicéron pût amasser des tonnes de documents incriminant Verrès. Selon Fuhrmann, lorsque le procès débuta le 5 août 69, Cicéron prit d'assaut la cour et présenta la montagne de preuves qu'il avait amassées. À l'aide de témoignages et de documents, Cicéron démontra comment Verrès avait extorqué 40 millions de sesterces aux Siciliens durant son règne. ${ }^{25}$ Verrès fût trouvé coupable et dût rembourser 3 millions de sesterces ${ }^{26}$, une bagatelle considérant le montant qu'il avait été accusé de voler.

Cependant, Murell fait un bon point lorsqu'il écrit que nous ne devrions pas prendre Verrès comme un exemple typique de gouverneur tout comme il fait valoir que Cicéron n'était pas un homme politique typique. ${ }^{27}$ Maintenant que nous avons vu cet exemple précis, nous pouvons maintenant nous permettre d'extrapoler et d'examiner la corruption dans un sens plus large.

\section{La République détruite}

C’est en 27 avant J. C. que le « château de cartes » que fût la République, s'effondre. Mais, ceci n'est pas un phénomène instantané. Les problèmes qui ont menés à la déchéance du système politique républicain sévissaient depuis longtemps, trop longtemps même.

\section{Déclin de la République}

Examinons premièrement, certains problèmes économiques. Selon Valode «l'effondrement des cours du blé, l'endettement des petits propriétaires et l'afflux massif d'esclaves... sont autant d'éléments qui perturbent l'ordre républicain. » Et il rajoute plus

\footnotetext{
${ }^{22}$ Murell, John. Cicero and the Roman Republic. Cambridge, U.K.: Cambridge University Press, 2008, 36. Aussi retrouvé dans le texte de Lòpez.

23 Idem, 43 et 37.

${ }^{24}$ Fuhrmann, Manfred. Cicero and the Roman Republic translated by W.E. Yuill. Cambridge Mass.: Blackwell

Publishers Ltd., 1995, 42.

25 Murell, Cicero and the Roman Republic, 43.

${ }^{26}$ Fuhrmann, Cicero and the Roman Republic, 44.

27 Idem, 42.
} 
loin comment les paysans, ayant perdu leurs terres et donc leurs emplois, viennent s'installer à Rome, ${ }^{28}$ créant ainsi un exode rural massif bien qu'il n'y ait pas plus de travail en ville.

Clerici et Olivesi rajoute que «Rome ne possède pas les instruments de crédit et les aptitudes financières nécessaires pour faire face aux transferts massifs de capitaux mobiliers orientaux en Italie; les prêts consentis par les chevaliers aux dynastes d'Orient qui doivent payer le tribut ne sont pas remboursés. Des banqueroutes secouent le monde financier et annoncent des faillites plus retentissantes.» ${ }^{29} \mathrm{Il} \mathrm{s}^{\prime}$ agit donc d'un amoncellement de problèmes économiques qui coulent, de plus en plus rapidement, la République. Valode décrit aussi comment certaines réformes agraires visant à redonner la terre aux plus pauvres (prisent à eux par les nobles) et à rendre la distribution du blé aux pauvres plus faciles, rencontrent une opposition des plus fortes de la part du Sénat (composé des nobles concernés par les réformes). ${ }^{30}$ Ceci accentue encore plus les tensions entre les classes.

Les problèmes économiques de la République ont des liens très étroits avec les problèmes politiques. Nous avons déjà montré comment certaines réformes agraires que l'on avait essayés d'implanter avaient exacerbés les tensions entres nobles et la population générale. Puis vinrent les guerres civiles. Plusieurs soulèvements populaires frappèrent la République à partir des années 110. Marius et Sylla et leur camp respectif (plébéiens et nobles) se livreront bataille. Certains peuples italiens recherchaient la citoyenneté romaine. Ils constitueront un gouvernement et se battront de (91 à 88) sans merci contre Rome. ${ }^{31}$ Un certain Sertorius essayera d'assurer la sécession de l'Espagne de 80 à72.32 Spartacus conduira une révolution d'esclaves (de 73 à 71), réprimée brutalement dans le sang. ${ }^{33}$ Ces révoltes ainsi que des guerres extérieures (contre Mithridate, les pirates siciliens, contre Jugurtha, les Teutons, etc) vont permettre à certains hommes de se faire un nom afin de briguer des positions politique : Sylla, Marius, Pompée, César.

Marius, fort de ces victoires contre Jugurtha, les Teutons et les Cimbres, deviendra le consul (107 et réélu sept ans de suite) du parti populaire et des plébéiens. Sylla (qui écrasera les Italiens), quant à lui sera élu consul en 88-87, opposera Marius et défendra plutôt le Sénat. Les plébéiens verront plusieurs améliorations durant les consulats de Marius (loi frumentaire qui fait en sorte que le blé est distribué aux pauvres à prix réduits, accès à l'armée) mais seront finalement réprimés par le Sénat en l'an 100. Sylla partira se battre contre Mithridate en 87 et à son retour en 83, une deuxième guerre civile est provoquée entre les nobles et les plébéiens. Sylla écrasera ce mouvement (aidé de Pompée) et sera nommé dictateur. Il procèdera à renforcer le Sénat, à miner le pouvoir des plébéiens et accroître son pouvoir en tuant ses adversaires politiques. ${ }^{34}$

\footnotetext{
28 Valode, Philippe. La Rome antique. Paris, France : Éditions De Vecchi, 2007, 44.

${ }^{29}$ Clerici, André et Olivesi, Antoine. La République romaine. Paris, France : Presses Universitaires de France, 1965 , 120.

30 Valode, La Rome antique, 46.

31 Idem, 48.

32 Idem, 50.

${ }^{33}$ Cébeillac-Gervasconi, Mireille. "Chapitre 15 - De la mort de Sylla au triomphe d'Octavien." Dans Histoire

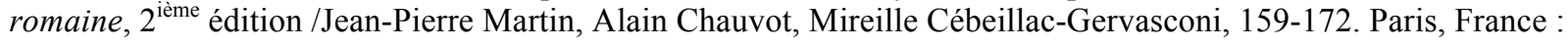
Armand Colin, 2010, 161-162.

34 Valode, Philippe. La Rome antique. Paris, France : Éditions De Vecchi, 2007, 44-50.
} 
Pompée, un fidèle de Sylla, réprimera la révolution de Sertorius ainsi que celle de Spartacus. Il parviendra à postuler pour le consulat en 70 , supporté par César. ${ }^{35}$ Pourtant, tout n'est pas rose. Le Sénat malmène Pompée, refuse d'octroyer des terres pour que s'y installent ses vétérans et s'oppose à lui. Pompée finit par renier les changements de Sylla et supporte ouvertement les populares (plébéiens et autres). ${ }^{36}$ En effet, lui et Crassus, l'autre consul du temps, entreprendront des réformes aboutissant à des mesures qui démantèleront les changements apportés par Sylla. ${ }^{37}$

César se fera une réputation d'homme politique et de leader militaire extraordinaire. Il sera tour à tour tribun militaire, édile, préteur, questeur et consul. Ces conquêtes militaires (notamment de la Gaule) feront en sorte que son génie militaire sera reconnu. En 59-60, Pompée, Crassus et César s'unissent sous le triumvirat. En 59, César, de nouveau consul (non sans une élection des plus difficiles car certains sénateurs auraient eu recours à des pots-de-vin afin d'empêcher son élection), implante de nouvelles mesures afin de limiter le pouvoir du Sénat et se rend maître de la Gaule où il trouvera toutes les composantes afin de prendre le pouvoir quelques années plus tard. ${ }^{38}$

En 52, Rome, assiégée par les optimates (défenseurs de l'oligarchie), n’a d'autre choix que de se tourner vers Pompée. Le Sénat l'instaure comme consul et lui donne les pleins pouvoirs. Le Sénat ordonne aussi à César de se destituer de ses fonctions. Pompée devient donc l'adversaire de César. Ce dernier, toujours posté en Gaule, se retournera contre son ancien allié et fort de ses quatre légions et des richesses obtenues en Gaule, franchira la frontière romaine pour envahir Rome. Pompée sera défait à Pharsale et César deviendra le maître incontesté de Rome ${ }^{39}$ César procédera à concentrer tous les pouvoirs en sa position. Il se déclare dictateur à vie en 44 (auparavant, il avait pris différents titres), étend son imperium sur tout l'empire romain, il se met à désigner les magistrats et les soumet à son autorité, transforme le Sénat en conseil et y place ses fidèles. Autant de réformes qui ne font qu'accroître son pouvoir. ${ }^{40}$ César sera assassiné en 44 mais ceci ne mettra aucunement fin aux ambitions monarchiques/impériales de certains.

Nous avons donc, ci-dessus, quelques facteurs du déclin républicain. Il est maintenant temps de regarder quel a été le rôle de la corruption dans ce déclin.

Rôle de la corruption

Étant donné toutes les causes (politiques, sociales, économiques) de l'écroulement de la République, nous ne pouvons pas assigner à la corruption un rôle central dans cet effondrement

\footnotetext{
35 Idem, 52.

${ }^{36}$ Cébeillac-Gervasconi, Mireille. "Chapitre 15 - De la mort de Sylla au triomphe d'Octavien." Dans Histoire romaine, $2^{\text {ième }}$ édition /Jean-Pierre Martin, Alain Chauvot, Mireille Cébeillac-Gervasconi , 159-172. Paris, France : Armand Colin, 2010, 52.

37 Bats, Maria. "Chapitre 3 - Rome de la fin de la Deuxième Guerre punique à la mort de César." Dans Le monde romain : histoire ancienne, sous la direction de M. Kaplan, 77-116. Rosny, France: Bréal, 1995, 89.

38 Idem, 89.

39 Valode, La Rome antique, 62-64 et Bats, "Chapitre 3 - Rome de la fin de la Deuxième Guerre punique à la mort de César", 89-90.

${ }^{40}$ Bats, Maria. "Chapitre 3 - Rome de la fin de la Deuxième Guerre punique à la mort de César." Dans Le monde romain : histoire ancienne, sous la direction de M. Kaplan, 77-116. Rosny, France: Bréal, 1995, 90.
} 
comme le démontre Lòpez. ${ }^{41}$ Mais, on ne peut ignorer la corruption et ses tentacules. En effet, Lòpez écrit «...elle [la corruption] a aidé certains hommes politiques romains à disposer d'un pouvoir supérieur à celui de leurs pairs.» ${ }^{42}$ Nous avons vu à quel point les provinces étaient importantes à l'enrichissement des gouverneurs. L'exemple de Verrès est le plus poignant de ce fait. Mais, il ne fût pas le seul.

Les expansions romaines en Espagne, en Gaule et autre ont permis aux dirigeants militaires d'accroître leur richesse ainsi que leur réputation. Bats décrit l'importance de Pompée au triumvirat selon sa richesse et son prestige ${ }^{43}$ et Lòpez démontre comment César «fonda toute sa carrière sur les ressources puisées dans les provinces, d'abord en Hispanie ultérieure, puis en Gaule, qui lui permirent d'influencer la politique, malgré son absence de Rome.»> ${ }^{44}$ L'auteur va même plus loin en écrivant aux mêmes pages que les imperia extraordinaria, ces imperiums extraordinaires que Pompée et César reçurent afin d'aller en campagne dans les provinces, servirent aux généraux afin de piller leurs provinces et de soumettre les légions dont ils disposaient à leur contrôle. Ainsi, nous avons donc les pièces qui menèrent à la guerre civile entre Pompée et César.

Lòpez écrit aussi que Pompée et César, lors de leurs consulats respectifs, mirent en place des lois afin de limiter les chances que les autres sénateurs fassent le même parcours qu'eux, faisant en sorte que le pouvoir resterait entre les mains des deux généraux. L'élection de César au consulat fût aussi très mouvementée comme il a été mentionné plus haut. Lòpez décrit comment César approcha un autre candidat afin qu'il donne des pots-de-vin pour qu'il s'assure que deux autres adversaire se retire de la course. Les sénateurs eurent vent de ceci et utilisèrent eux-mêmes de pots-de-vin afin de garantir que César ne soit pas élu avec un partenaire serviable mais plutôt avec un opposant. ${ }^{45}$

En guise de conclusion, une récapitulation. Nous avons abordé premièrement l'organisation et le fonctionnement politique romain typique à l'époque de la République et de Cicéron. Ensuite, est venu une discussion de ce qu'était exactement la corruption, sa définition chez les romains, ses différentes formes et où elle s'insérait dans la politique républicaine. Ceci fût fait afin de donner un contexte au lecteur. Puis, pour terminer nous en sommes venus aux problèmes qui sévissaient à la fin de la République et aux effets de la dite corruption. Nous avons vu un survol assez bref de tous ces éléments car chacun de ceux-ci pourraient constituer une dissertation en eux-mêmes. Nous espérons toutefois que le survol a présenté un portrait assez complet de ce que nous voulions aborder.

La corruption existe toujours aujourd'hui et elle ne démordra pas de si tôt. Nous n'avons qu'à regarder ce qui se passe au Québec avec les allégations de corruption dans le milieu de la construction. Certains hommes politiques québécois auraient reçus des «enveloppes brunes», un euphémisme risible employé pour désigner les pots-de-vin. La mafia italienne et certains entrepreneurs en construction auraient fait usage de ces pots-de-vin afin de s'assurer que plusieurs projets de construction situé à Montréal et ailleurs au Québec leurs reviennent. Ces mêmes entrepreneurs se seraient mis en accord afin de décider qui d'entre eux recevraient le droit

\footnotetext{
41 Rosillo López, Cristina. La corruption à la fin de la République romaine (IIe-Ier s. av. J.-C.) : Aspects politiques et financiers. Stuttgart, Allemagne : Franz Steiner Verlag, 2010, 236.

42 Idem, 236.

43 Bats, "Chapitre 3 - Rome de la fin de la Deuxième Guerre punique à la mort de César", 89.

44 Rosillo López, La corruption à la fin de la République romaine (IIe-Ier s. av. J.-C.) : Aspects politiques et financiers, 235-236.

45 Idem, 185.
} 
de soumissionner pour un contrat et à combien ces soumissions se chiffreraient. Il va sans dire que les coûts furent gonflés artificiellement et finirent par coûter plusieurs dizaines millions de dollars aux contribuables du Québec.

Une commission d'enquête provinciale fût mise sur pied afin de faire la lumière sur le phénomène. Celle-ci est toujours en cours en date du 19 décembre 2012. Une unité spéciale a aussi été créé (Unité permanente anticorruption ou UPAC) afin d'enquêter les malversations et les mauvaises gestions de fonds publics par certains maires et hommes politiques du Québec. À titre d'exemple les maires de Montréal, de Laval, de Mascouche et de Saint-Rémi ont tous démissionné ou été démis de leurs fonctions à la suite d'accusations de corruption. De plus, la tombée du gouvernement libéral québécois en septembre 2012 a aussi certaines de ses racines dans les accusations de corruption et de collusion qui sont apportés depuis quelques années.

L'exemple ci-dessus n'en est qu'un parmi plusieurs. Pourtant, nous voyons que la corruption est un phénomène encore très présent, très grave et très nuisible. Elle gruge les fonds monétaires publics, détruit la confiance des contribuables envers les institutions gouvernementales et peut amener des bouleversements quant aux partis politiques au pouvoir. Ainsi, la corruption menace la stabilité économique, politique et sociale des villes, des régions, des provinces et des pays. Mais, nous croyons qu'elle est inévitable tant et aussi longtemps que la cupidité, l'égoïsme et la quête du pouvoir règneront.

\section{Bibliographie :}

\section{Livres}

Clerici, André et Olivesi, Antoine. La République romaine. Paris, France : Presses Universitaires de France, 1965.

Combès, Robert. La République à Rome (509-29 av. J.-C.). Paris, France : Presses Universitaires de France, 1972.

Fuhrmann, Manfred. Cicero and the Roman Republic translated by W.E. Yuill. Cambridge Mass.: Blackwell Publishers Ltd., 1995.

Grimal, Pierre. La civilisation romaine. Paris, France: Les Éditions Arthaud, 1984.

Lacey, W. K. Cicero and the end of the Roman Republic. Londres, U.K.: Hodder and Stoughton, 1978.

Murell, John. Cicero and the Roman Republic. Cambridge, U.K.: Cambridge University Press, 2008 .

Rosillo López, Cristina. La corruption à la fin de la République romaine (IIe-Ier s. av. J.-C.) : Aspects politiques et financiers. Stuttgart, Allemagne : Franz Steiner Verlag, 2010.

Valode, Philippe. La Rome antique. Paris, France : Éditions De Vecchi, 2007.

Notes de cours 
Maillet-Rao, Caroline. «La royauté et la république romaine» Science Sociales 311, Automne 2011.

Chapitres de livres

Bats, Maria. "Chapitre 3 - Rome de la fin de la Deuxième Guerre punique à la mort de César." Dans Le monde romain : histoire ancienne, sous la direction de M. Kaplan, 77-116. Rosny, France: Bréal, 1995.

Cébeillac-Gervasconi, Mireille. "Chapitre 15 - De la mort de Sylla au triomphe d'Octavien."

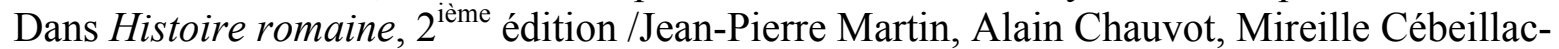
Gervasconi , 159-172. Paris, France : Armand Colin, 2010.

Gaillard, Jacques. "Les rituels politiques de la République." Dans Rome ${ }^{\text {er }}$ siècle av. J.-C. Ainsi périt la République des vertus..., dirigé par Jacques Gaillard, 42-57. Paris, France : Les Éditions Autrement, 1996.

Klein, Bernard. "Chapitre 1 - Rome, des origines à la fin du IIIe siècle." Dans Le monde romain : histoire ancienne, sous la direction de M. Kaplan, 11-46. Rosny, France: Bréal, 1995. 